\title{
"Si llevas el Anillo conéctate a la Web": Análisis de las opiniones encontradas en foros de Internet sobre el uso del Anillo Anticonceptivo Vaginal
}

\author{
M. Candelaria Ayuso Rayaa , M. Aránzazu Romero Cebriánª, Juan Miguel Armero Simarroa, \\ María Arias Alaminos ${ }^{a}$, Nieves Pérez López ${ }^{b}$, Francisco Escobar Rabadán ${ }^{c}$
}

\begin{abstract}
${ }^{a}$ Residente de Medicina Familiar y Comunitaria. Unidad Docente de Albacete.

b Diplomada Universitaria en Enfermería. Centro de Salud Universitario Zona IV de Albacete.
\end{abstract}

c Médico de Familia. Centro de Salud Universitario Zona IV de Albacete. Profesor Asociado de la Facultad de Medicina de Castilla-La Mancha.

\section{Correspondencia: Candelaria} Ayuso Raya, Centro de Salud Zona IV, Calle Seminario $n^{\circ} 4$, 02006-Albacete, España. Telf.: 967510094, fax: 967507362 , e-mail:

candeayuso@hotmail.com.

Recibido el 16 de octubre de 2008.

Aceptado para su publicación el 25 de noviembre de 2008.

\section{RESUMEN}

Objetivos. Conocer las opiniones acerca del uso del anillo anticonceptivo vaginal expresadas en foros de Internet.

Diseño del estudio. Estudio cualitativo.

Emplazamiento. Atención comunitaria.

Participantes. Participantes en foros de Internet relacionados con el uso del anillo vaginal.

Mediciones y Resultados. Por medio de una búsqueda en Google se identificaron foros en los que se hiciera referencia al uso del anillo vaginal. Se han revisado 85 foros, seleccionando opiniones de 246 participantes. Seguidamente se clasificó y sintetizó la información recogida.

Como ventajas más relevantes se han destacado: comodidad de administración, no suele haber olvidos, regula el ciclo menstrual, no produce aumento de peso, su eficacia no se afecta por vómitos o diarreas, y se evita el primer paso de metabolismo hepático. Entre los inconvenientes habría que resaltar: sensación de que se va a salir, precio, algunas mujeres se quejan de irregularidad menstrual, irritación vaginal, sensibilidad mamaria, aumento de peso, cambios de humor, cefalea, menos flujo vaginal, náuseas, cansancio, e interacciones medicamentosas. Se subrayan también inconvenientes en relación con la aplicación del dispositivo, el modo de conservación o cuándo empieza a ser efectivo. Las opiniones sobre cómo afecta a las relaciones sexuales oscilan entre quienes consideran que aumenta la libido y quienes refieren una disminución del deseo sexual, o bien molestias en las relaciones, debido a que la pareja o las usuarias lo notan.

Conclusiones. El anillo vaginal es un método anticonceptivo fácil de utilizar, cómodo de aplicar y seguro, sin embargo, presenta frecuentes efectos adversos sobre todo a nivel local.

Palabras clave. Dispositivos Anticonceptivos Femeninos. Satisfacción Personal. Internet.

\section{ABSTRACT}

"If you use the Ring get on line": Analysis of the opinions on the use of the Vaginal Contraceptive Ring found on Internet forums

Objectives. To learn the opinions on the use of the vaginal contraceptive ring expressed on Internet forums.

Study design. Qualitative study

Setting. Community healthcare.

Participants. Participants on internet forums related to the use of vaginal rings.

Measurements and Results. A search on Google identified forums that referred to the use of the vaginal ring. A total of 85 forums were reviewed, and opinions from 246 participants were selected. The information was then classified and summarised.

The most significant advantages pointed out were: easy to use, not easily forgotten, regulates menstrual cycle, doesn't cause weight gain, its efficacy is not affected by vomiting or diarrhoea, it avoids first pass hepatic metabolism. Amongst the disadvantages pointed out were: feeling that it is going to slip out, some women complained of irregular periods, vaginal irritation, breast tenderness, weight gain, nausea, mood swings, headache, vaginal dryness, nausea, tiredness and drug interactions. Other drawbacks related to the insertion of the ring, how to store it or when it becomes effective, were emphasised. Opinions on how it affected sexual relations ranged from increased libido to a decrease in sexual desire, or the user or user's partner can feel it during sexual intercourse.

Conclusions. The vaginal ring is an easy to use contraceptive method, easy to insert and safe. However there are frequent adverse effects especially local effects.

Key words. Contraceptive Devices. Female. Personal Satisfaction. Internet.

Una versión preliminar de este trabajo fue presentada como comunicación oral en el $9^{\circ}$ Congreso de Atención Primaria de Castilla-La Mancha, celebrado los días 5, 6 y 7 de junio de 2008 en Guadalajara. 


\section{INTRODUCCIÓN}

Los intentos de controlar la fertilidad son tan antiguos como la propia humanidad. La aparición de la anticoncepción tuvo que esperar hasta el siglo XIX, en el que se ideó el preservativo. El primer anticonceptivo hormonal aprobado por la FDA fue el Enovid 10 (10 $\mathrm{mg}$ de noretinodel $+0.015 \mathrm{mg}$ de mestranol), en mayo de 1960. Según la Organización Mundial de la Salud, 60 millones de mujeres en el mundo utilizan anticonceptivos hormonales, lo que los sitúa como la cuarta opción anticonceptiva, después del coitus interruptus, el preservativo y el dispositivo intrauterino ${ }^{1}$. En España, según una encuesta publicada por el equipo Daphne $^{2}$ en 2007, hay un total de 10 millones de mujeres en edad fértil, de las que únicamente el $79.8 \%$ utilizan algún método anticonceptivo.

El anillo anticonceptivo vaginal se encuentra disponible desde el año 2003. Consiste en un anillo de plástico blando con un diámetro exterior de $5,4 \mathrm{~cm}$. Libera $15 \mathrm{mcg}$ de etinilestradiol y $120 \mathrm{mcg}$ de etonogestrel cada 24 horas, inhibiendo la ovulación. Estas hormonas pasan al organismo a través de la pared vaginal, lo que permite una menor dosis que por vía oral. La mujer se lo inserta fácilmente dentro de la vagina y una vez insertado se deja colocado durante 3 semanas, y posteriormente la mujer se retira el anillo fácilmente. A continuación se ha de insertar un nuevo anillo exactamente una semana después de retirado el anterior ${ }^{3,4}$. Ha demostrado mejor aceptación que otros métodos anticonceptivos nuevos, como los parches trasdérmicos ${ }^{5}$.

Nuestro estudio nace de la consulta de atención primaria, cuando una de nuestras pacientes solicitó consejo anticonceptivo. Tras conocer sus preferencias se eligió como método el anillo vaginal. Tras varias semanas de uso comenzó a consultar por vaginitis de repetición por hongos. Sin embargo, en este intervalo de tiempo la paciente había optado, tras revisar varios foros en Internet, por la aplicación de pomada tópica, tal y como comentaban otras usuarias. Este hecho llamó nuestra atención, preguntándonos sobre el contenido de la información a la que podrían estar teniendo acceso nuestras pacientes a través de la red.

Así, nos planteamos como objetivo conocer las opiniones de las usuarias sobre la utilización del anillo anticonceptivo vaginal a través de foros de Internet.

\section{MATERIAL Y MÉTODO}

Se trata de un estudio cualitativo, realizado entre los meses de marzo y mayo de 2008. Por medio de una búsqueda en Google se identificaron foros en los que se hiciera referencia al uso del anillo vaginal, desde su comercialización en España hasta el año 2008. Optamos por analizar la información disponible en castellano, seleccionando en un primer proceso foros, y posteriormente opiniones, evitando la inclusión de temas que no fuesen derivados del uso del anillo. Se decidió no incluir la identidad de las participantes, por motivos de confidencialidad.

Posteriormente, tras consensuar los criterios de selección, agrupamos las opiniones bajo epígrafes, lo que permitió homogeneizar criterios. Finalmente, se realizó una labor de clasificación y síntesis de la información recogida.

\section{RESULTADOS}

Se han revisado 85 foros, seleccionando opiniones de 246 participantes. La mayoría de las opiniones registradas están en relación con las ventajas en la utilización y dudas en relación con la efectividad.

Las ventajas más relevantes que se han destacado incluyen: comodidad de administración, no suele haber olvidos, regula el ciclo menstrual, no produce aumento de peso, su eficacia no se afecta por vómitos o diarreas y se evita el primer paso de metabolismo hepático, disminuye el vello corporal, e incluso algunas usuarias refieren mayor deseo sexual.

Como inconvenientes habría que resaltar: la sensación de que se va a salir, el precio, algunas mujeres se quejan de irregularidad menstrual, efectos secundarios frecuentes como irritación vaginal, prurito y vaginitis, sensibilidad mamaria, aumento de peso, irritabilidad y cambio de humor, cefalea, menos flujo vaginal, náuseas, cansancio, e interacciones medicamentosas. Se subrayan también algunos inconvenientes en relación con la aplicación del dispositivo, el modo de conservación o cuándo empieza a ser efectivo.

Las opiniones sobre cómo afecta a las relaciones sexuales oscilan entre quienes consideran que aumenta la libido y quienes refieren una disminución del deseo sexual, o bien molestias en las relaciones, debido a que la pareja o las usuarias lo notan. El no ser un método de barrera también lo tienen en cuenta, ya que no previene de las ETS.

En la tabla 1 presentamos las opiniones que nos han resultado más llamativas, encuadradas en los epígrafes que consideramos de mayor relevancia.

\section{DISCUSIÓN}

Nuestro estudio se planteó desde una gran curiosidad acerca de la información que podríamos encontrar, sorprendidos por una paciente altamente sensibiliza- 
Comodidad de administración

Menos olvidos

Regularidad del ciclo menstrual

Aumento de libido y mejora de relaciones

Peso

Absorción

Metabolismo

Sensación de que se va a salir

Precio

No barrera

Molestias locales, irritación, vaginitis

Sensibilidad mamaria

Irritabilidad, cambios de humor

Acné

Cefalea

Retención de líquidos

Menos flujo vaginal

Menstruación

Náuseas

Antibióticos

Drogas

Conservación

Retirada temporal

Calendario de administración Efectividad

Cómo influye la colocación

Cambio de método

Disminución de la libido

Lo notan

Molestias en las relaciones

Uso con otros dispositivos

Enfermedades vasculares
"A mí me resulta cómodo ponérmelo y quitármelo"

"No interfiere en mi ritmo de vida, puedo estar tranquila de olvidos, paso de tener que llevarme en mis viajes pastillas o parches"

"Me viene muy poquita regla y ni siquiera me duele y no me he hinchado"

"No te corta el rollo, no tienes miedo a que se rompa y te olvidas durante tres semanas"

"Me noto menos pesada, más ágil y menos hinchada"

"Si tienes vómitos o diarrea no pasa nada porque en ningún momento se ve afectada la absorción"

"No pasa por el hígado al no ingerirse como la mayoría de los medicamentos"

"Cada cierto rato tengo que empujar el dichoso anillito dentro porque se me sale, lo noto al sentarme,

al andar... Continuamente lo noto ahí, con la sensación de que si hago un movimiento brusco se me va

a caer... Luchando por salir"

"Merece la pena aunque sean quince euros... El preservativo es un coñazo"

"Es un anticonceptivo hormonal y no de barrera, con lo que no previene de ETS"

"Lo más molesto son los picores, ellos lo ponen con los efectos secundarios raros pero lo tendrían que poner en la columna de comunes, porque todo el mundo que conozco que lo usa, los ha sufrido"

"Durante las tres semanas que lo utilizas tienes los pechos más grandes (algo bueno para mí) y no notas nada, es genial"

"Tengo cambios de humor, hipersensibilidad e irritabilidad. En definitiva, para mí es como vivir en una pre-regla constante"

"Mi cara más libre de impurezas"

"Me duele bastante la cabeza, llevo como cinco días con dolor constante aunque también he de decir que es soportable"

"Retenía muchos líquidos y se me hinchaban las piernas y dejé de utilizarlo"

"El único inconveniente es que como efecto secundario provoca sequedad vaginal, pero existen productos que lo solucionan"

"Me dura un poco más que con la píldora, y si con la píldora no tenía dolores, con el anillo sí"

"Tengo pequeñas pérdidas cada dos o tres meses. Hasta una semana antes de que me correspondiera la regla empiezo a manchar, se debe a que el anillo tiene poca dosis y no me regula el ciclo completamente"

"Puede presentarse leve dolor de cabeza y náuseas que se regularizan al segundo ciclo"

"He usado el anillo hasta quedarme embarazada y cuando tomas antibióticos reduce su efecto como cualquier otro contraceptivo"

"Quería preguntaros a ver si sabéis si las drogas alteran el efecto del anillo, sobre todo los porros"

"No hace falta que lo lleves en una nevera mientras la temperatura sea menos de treinta grados, ya que a partir de esa temperatura es cuando empieza a salir la hormona del anillo. De todas formas, si lo metéis de frío en hojas de periódico, el frío se mantiene bastante bien"

"Si se te cae es lavarlo y para adentro de nuevo, si pasan tres horas tienes que ponerte uno nuevo"

"Estás con él puesto tres semanas y la cuarta semana lo retiras y es cuando te viene la regla"

"Es altamente efectivo y según los expertos más que el condón, pues en nuestro caso no hemos tenido aún ningún susto"

"Si lo colocas mal puedes notarlo dentro, pero lo recolocas y ya está"

"Si no usabas píldora se pone el día 1 del ciclo (el día que te viene la regla). También se puede poner hasta el día 5 y usar preservativo 1 semana. Si tomabas píldora lo pones el día que se acabe tu semana de descanso"

"Es muy útil pero se me quitan las ganas de sexo"

"Mi chico sí que lo nota, de hecho yo me lo quito para tener relaciones porque él lo nota y le da cosa"

"Al mantener relaciones sexuales noto molestias, no siempre, pero de vez en cuando sí, es como si hiciese tope y se mi novio intenta forzar un poco duele. Añado que no tenemos relaciones con posturas extrañas"

"Cuando usé por primera vez el anillo, ya usaba la Mooncup, esperé al quinto día de la regla, tuve precauciones durante una semana y al ciclo siguiente ya no coincidieron mooncup y anillo" "Puedes seguir usando tampax al mismo tiempo"

"Al igual que otros anticonceptivos hormonales combinados aumenta el riesgo de trombosis si tienes sobrepeso"

"Está contraindicado en muchos casos, por ejemplo fumadoras, ya que favorece las trombosis"

Tabla 1. Opiniones de participantes en foros de Internet sobre el uso del anillo vaginal. 
da con las opiniones no profesionales brindadas a través de foros de Internet. No nos vimos defraudados dadas la importancia y trascendencia de las opiniones y comentarios sobre las posibles consecuencias derivadas del uso del anillo anticonceptivo vaginal.

No hemos encontrado ningún estudio similar al nuestro. Este nos ha permitido conocer la opinión de un gran número de usuarias, de forma que hemos podido aproximarnos a la situación real de personas que escriben sus opiniones acerca de los factores asociados al cumplimiento, a las alteraciones menstruales, a la percepción personal, dudas acerca de efectos adversos, efectividad, opiniones sobre la esfera sexual, aceptación del método derivado del uso individual, y en definitiva a las repercusiones en su salud.

Podríamos asegurar que para la mayoría de las usuarias el anillo es fácil de utilizar. Comparado con otros métodos anticonceptivos, valoran muy positivamente su aplicación durante 3 semanas, así como el evitar la vía oral. Su efecto adverso más frecuente es el derivado de su uso a nivel local, mientras que otros, como las náuseas, cefalea o tensión mamaria, las usuarias reconocen que son poco frecuentes y desaparecen al cabo de unas semanas de su utilización, pero que en ningún caso condicionan el cambio de método. Para algunas mujeres la dosis de estrógenos afectó inicialmente a su estado de ánimo de forma negativa, pero para la mayoría influyó de forma positiva al sentirse protegidas, más lubricadas, y en caso de salida accidental ninguna pareja lo consideró motivo para cambio en el método.

La gran mayoría de las opiniones encontradas en los foros están en consonancia con la literatura médica. Así, la opinión sobre la alta efectividad y la buena tolerancia del método coincide con los hallazgos de Weisberg et $\mathrm{al}^{6}$, que demostraron que el anillo vaginal es eficaz, en general bien tolerado, aunque causa con frecuencia relativamente alta náuseas transitorias después de la inserción de un nuevo anillo, probablemente relacionado con acumulación de etinilestradiol. En un trabajo posterior ${ }^{7}$ confirmaron la eficacia anticonceptiva en relación con el mantenimiento sérico de adecuados niveles de estrógenos, así como la satisfacción en cuanto a su uso.

La satisfacción con el método se confirma en el estudio de Schafer et $\mathrm{al}^{8}$, en el que un $61 \%$ de usuarias del anillo se mostraban muy satisfechas, frente al $34 \%$ de mujeres que tomaron anticonceptivos orales a dosis bajas.

En cuanto a los síntomas vaginales, Veres et al ${ }^{9}$ analizaron la presentación de estas complicaciones en pacientes tratadas con el anillo vaginal, comparadas con mujeres tratadas con anticonceptivos orales, a través de la existencia de signos y síntomas, así como exámenes de laboratorio. Observaron un incremento de la humedad vaginal en mujeres que usaban el anillo, sin significado patológico, y un incremento de la concentración de lactobacillus productores de $\mathrm{H}_{2} \mathrm{O}_{2}$, con efecto beneficioso en la vagina, al prevenir infecciones víricas y bacterianas y estar inversamente asociada con vaginosis bacteriana.

Las opiniones encontradas en nuestro estudio en relación con la menstruación son muy variables, dependiendo de la experiencia personal de cada usuaria. Westhoff et $\mathrm{al}^{10}$ compararon los patrones de sangrado (registro en un diario tanto de sangrados menstruales como de spotting) en los 84 días siguientes al inicio de un tratamiento inmediato (Quick Start) con un anticonceptivo hormonal oral frente al anillo vaginal, encontrando diferencias significativas en los patrones de sangrado a lo largo de los 3 ciclos entre ambos grupos. Las mujeres que seguían tratamiento oral informaban resultados de sangrado más adversos que las asignadas al anillo. La diferencia en el número de días de sangrado-spotting entre las usuarias de los dos métodos es sobre todo atribuible a la diferencia en spotting, ya que las usuarias de las pastillas tenían más. Atribuyen los mejores patrones de sangrado con menos dosis de estrógeno (15 mcg/día del anillo frente a 25 mcg/día de las pastillas) al hecho de evitar las fluctuaciones comunes a la medicación oral. Por otra parte, Bjarnadóttir et $\mathrm{al}^{11}$ demostraron una incidencia de sangrados irregulares en mujeres tratadas con aniIlo anticonceptivo vaginal inferior al $5 \%$, significativamente inferior al que presentaban mujeres tratadas con contraceptivos orales.

En concordancia con alguno de los estudios anteriores, Ahrendt et $\mathrm{al}^{12}$, que estudiaron a 983 mujeres a lo largo de 13 ciclos en un ensayo aleatorizado multicéntrico, observaron un alto cumplimiento, tanto para usuarias del anillo $(89,2 \%)$ como para las de anticonceptivos por vía oral (85,5\%). Los efectos adversos más frecuentes fueron locales en el caso del anillo, frente a los estrógeno-dependientes con el uso de la vía oral. Roumen ${ }^{13}$, en una revisión de ensayos clínicos aleatorizados, concluyó que el anillo vaginal tiene la misma eficacia que los anticonceptivos orales combinados con menor exposición sistémica. Mantienen adecuadamente los niveles séricos de estrógenos y logran un mejor control del ciclo. Sin embargo, presentan más efectos locales en relación con tasas de estrógenos más altas en epitelio vaginal.

Como conclusión de nuestro estudio, hemos de subrayar la alta eficacia, tolerabilidad y aceptabilidad atribuida al anillo vaginal por las mujeres que partici- 
paban en los foros de Internet analizados. La mayoría consideraban la inserción y extracción del anillo fácil, y lo prefieren como método anticonceptivo frente a la vía oral, asegurando una menor tasa de olvidos en su uso. La incidencia de efectos adversos tales como la tensión mamaria, la cefalea, la disminución de la libido y las náuseas no son frecuentes en mujeres usuarias del anillo, pero sí lo son los efectos adversos locales.

Internet constituye una fuente de información que ofrece infinidad de recursos, con grandes potencialidades, y con importantes implicaciones si consideramos especialmente el ámbito de la salud. Adentrarnos en su conocimiento es un reto para los profesionales, en el que la metodología de investigación cualitativa constituye una herramienta valiosísima. En este sentido entendemos que se nos presenta un vastísimo campo de investigación.

\section{BIBLIOGRAFÍA}

1. Sánchez Borrego R, Martínez Pérez O. Guía Práctica en Anticoncepción Oral Basada en la Evidencia. Madrid: Emisa; 2003.

2. Encuesta Daphne. El cumplimiento en Anticoncepción Hormonal oral en España, 2007.

3. http://www.equipodaphne.es/archivos/encuestas/03_Encuesta \%20Anticoncepcion\%202007\%20rueda\%20de\%20prensa\% 20231007.pdf (con acceso el 11-9-2008).

4. Gallo MF, Grimes DA, Schulz KF. Parche transdérmico y anillo vaginal versus anticonceptivos orales combinados para la anticoncepción. La Biblioteca Cochrane Plus 2008: número 1.
5. Herndon EJ, Zieman M. New contraceptive options. Am Fam Physician 2004; 69:853-60.

6. Creinin MD, Meyn LA, Borgatta L, Barnhart K, Jensen J, Burke AE, et al. Multicenter comparison of the contraceptive ring and patch: a randomized controlled trial. Obstet Gynecol 2008; 111:267-77.

7. Weisberg E, Fraser IS, Lacarra M, Mishell DR, Jackanicz T. Effect of different insertion regimens on side effects with a combination contraceptive vaginal ring. Contraception 1997; 56:233-9.

8. Weisberg E, Fraser IS, Lacarra M, Mishell DR, Alvarez F, Brache V, Nash HA. Efficacy, bleeding patterns and side effects of a 1year contraceptive vaginal ring. Contraception 1999; 59:311-8.

9. Schafer JE, Osborne LM, Davis AR, Westhoff C. Acceptability and satisfaction using Quick Start with the contraceptive vaginal ring versus an oral contraceptive. Contraception 2006; 73:488-92.

10. Veres S, Miller L, Burington B. A comparison between the vaginal ring and oral contraceptives. Obstet Gynecol 2004; 104:555-63.

11. Westhoff C, Osborne LM, Schafer JE, Morroni C. Bleeding patterns after inmediate initiation of an oral compared with a vaginal hormonal contraceptive. Obstet Gynecol 2005; 106:89-96.

12. Bjarnadóttir RI, Tuppurainen M, Killick SR. Comparison of cycle control with a combined contraceptive vaginal ring and oral levonorgestrel/ethinyl estradiol. Am J Obstet Gynecol 2002; 186:389-95.

13. Ahrendt H, Nisand I, Bastianelli C, Gómez MA, Gemzell-Danielsson K, Urdl W, et al. Efficacy, acceptability and tolerability of the combined contraceptive ring, Nuvaring, compared with an oral contraceptive containing 30 microg of ethinyl estradiol and $3 \mathrm{mg}$ of drospirenone. Contraception 2006; 74:451-7.

14. Roumen FJME. The contraceptive vaginal ring compared with the combined oral contraceptive pill: a comprehensive review of randomized controlled trials. Contraception 2007; 75:420-9. 